\title{
Alfredo Lanari, un estilo de investigación clínica
}

\author{
Alfredo Lanari, a clinical research style
}

${ }^{1}$ Licenciada en Sociología. Doctora en Ciencias Sociales. Profesora de Sociología, Universidad de Buenos Aires (UBA). Profesora de Sociología Universidad Nacional de Quilmes (UNQ). Becaria posdoctoral, Consejo Nacional de Investigaciones Científicas y Técnicas (CONICET), Argentina.

laromero@unq.edu.ar

Romero, Lucía ${ }^{1}$

RESUMEN La institucionalización de la investigación clínica en la Argentina adquirió su máxima madurez a partir de la creación, en 1957, del Instituto de Investigaciones Médicas de la Facultad de Medicina de la Universidad de Buenos Aires, y del impulso de quien fuera su director durante casi 20 años, Alfredo Lanari. En este trabajo analizo las modalidades mediante las cuales esta figura construyó un estilo de investigación clínica y una posición de referente en la medicina local que le permitieron Ilevar adelante dicha realización institucional. Esta construcción fue fruto de una empresa personal pero al mismo tiempo abrevó en un contexto más amplio de transformaciones en la medicina mundial y en la Universidad de Buenos Aires. Esta investigación se llevó a cabo combinando el uso de fuentes orales (entrevistas a miembros del Instituto de Investigaciones Médicas, integrantes de la revista Medicina y de la Sociedad Argentina de Investigación Clínica) y documentales (legajos académicos y artículos científicos).

PALABRAS CLAVE Historia del Siglo XX; Medicina Clínica; Investigación Clínica; Argentina.

ABSTRACT The institutionalization of clinical research in Argentina reached its point of greatest maturity with the creation, in 1957, of the Institute of Medical Investigations (Instituto de Investigaciones Médicas) of the Faculty of Medicine of the Universidad de Buenos Aires, and the drive of the man who was its director for almost 20 years, Alfredo Lanari. In this paper I analyze the ways in which he generated a style of clinical research and a referential position in local medical field that allowed him to carry out said institutional realization. This achievement was the result of a personal enterprise and at the same time part of a larger context of transformations within the medical discipline worldwide and at the Universidad de Buenos Aires. This study was carried out combining oral and documentary sources, such as interviews with physicians at the Institute of Medical Investigations, members of the journal Medicina and of the Argentine Society of Clinical Investigation (Sociedad Argentina de Investigación Clínica), as well as academic files and scientific articles.

KEY WORDS History, 20th Century; Clinical Medicine; Biomedical Research; Argentina. 


\section{INTRODUCCIÓN}

El nombre de Alfredo Lanari indudablemente ocupa un lugar muy significativo en la historia científica y médica argentina de la segunda mitad del siglo XX. Su actividad profesional, caracterizada por un afán innovador, se plasmó en las diversas empresas científicas que emprendió con el objetivo de renovar diferentes aspectos de la medicina local y regional.

Esto impactó en la transformación de tres frentes fundamentales: en la educación médica, en la práctica asistencial y en la investigación. Así, su figura, entre otras personalidades médicas, estuvo detrás del impulso de las reformas de enseñanza de grado (unidad docente hospitalaria, impartiendo el ciclo clínico de tres años dentro del hospital) y postgrado (residencias médicas) introducidas e implementadas al promediar la década de 1960 en la Facultad de Medicina de la Universidad de Buenos Aires. Fue quien instaló la discusión de la dedicación full time del practicante, docente e investigador en el ámbito de la medicina clínica local y quien desempeñó por ello los mayores esfuerzos en hacer converger las tradiciones clínicas y experimentales.

Estos cambios se materializaron de forma paradigmática en el Instituto de Investigaciones Médicas (IIM) de la Facultad de Medicina de la Universidad de Buenos Aires (UBA), del cual Lanari fue director desde su creación en 1957 hasta el año 1976.

Sin restarle protagonismo a su intervención en el campo de la clínica médica local y en la creación del IIM, es imprescindible reconocer la injerencia que en él tuvo el clima de ideas y de acciones innovadoras ocurridas en el plano cosmopolita de la disciplina en los años de la segunda posguerra y en el clima político académico de renovación y profesionalización científica que vivió la universidad argentina a partir del derrocamiento del gobierno peronista en 1955 (1).

En el contexto disciplinar de la segunda posguerra, en los dos centros de referencia mundial de la investigación, organización y financiamiento médico, EE.UU. y Francia, ocurrieron transformaciones de envergadura en las instituciones estatales que tenían a su cargo la promoción y ejecución de tales actividades. El período entre 1955 y 1968 se conoció como "Ios años de oro" de la expansión presupuestaria y de la capacidad financiera del National Institute of Health (NIH) y, a su vez, con la transformación del Institut National d'Hygiène de Francia en el Institut National de la Santé et la Recherche Médicale (INSERM), en 1964, se concretó un nuevo entrelazamiento entre actividades de investigación, atención y docencia médica hospitalaria, a partir del cual comenzó a redefinirse el perfil profesional del investigador clínico y las relaciones entre las ciencias de laboratorio y la práctica clínica médica (2).

En el plano de la enseñanza médica, en la década de 1950 comenzaron a darse un conjunto de innovaciones que fueron fruto de un largo proceso a nivel mundial y regional, con fuertes repercusiones locales, de revisión y discusión sobre los modelos de educación médica prevalecientes. Se desarrollaron un conjunto de propuestas de reformas de la enseñanza médica en espacios tales como los Congresos de Educación Médica mundiales y regionales $(3,4)$, muchas de las cuales tendían a consolidar la "flexnerización" de la medicina. Este proceso había comenzado en la década de 1910 y consistió en el viraje de la clínica médica desde el paradigma anatomopatológico hacia el de las ciencias básicas (la fisiología) y de laboratorio, como parte de una serie de reformas producidas en la enseñanza médica de las universidades estadounidenses, a partir del denominado "informe Flexner" (5). Este informe fue el resultado del trabajo que la Carnegie Foundation le había encargado a Abraham Flexner sobre el estudio de la enseñanza de medicina en las universidades norteamericanas y en el cual se dejó asentada la importancia prioritaria del trabajo experimental, cultivado en el modelo germánico de universidad, que destacaba el fundamento científico de la medicina. Justamente este conformó el eje de su innovación: la consideración de la medicina apoyada en las ciencias básicas y, por ende, la importancia de estas en la formación del médico, dando contenido a la "medicina científi-

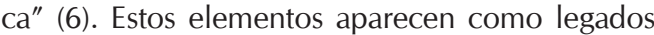
en la idea y práctica del full time médico que impulsó Lanari, así como también en su práctica médica integral de investigación, asistencia y docencia, que combinaba la investigación experimental con la asistencia clínica. 
En el plano local también existió un conjunto de ideas y acciones de sujetos enrolados en movimientos de cambios y renovación universitaria, con resonancias en la Facultad de Medicina de la UBA, que influyeron y retroalimentaron la orientación que tomó la participación de Lanari en la medicina clínica local.

La Universidad de Buenos Aires vivió a partir de 1955 un proceso de renovación cuya característica más destacada fue el inicio de la profesionalización de las actividades de investigación y docencia, es decir, la creación y ampliación de cargos ocupacionales full time en la universidad (1), que cobró un singular dinamismo a partir de la gestión de Risieri Frondizi al frente del rectorado de la UBA en 1957, así como también desde la Facultad de Ciencias Exactas y Naturales (FCEN), con el decanato de Rolando García (a) (9)

En la Facultad de Medicina se constituyó un frente modernizador con los profesores que orbitaban alrededor de la personalidad de Bernardo Houssay (b). Los puntos sobre los cuales se conformaron las mayores convergencias entre los contenidos centrales de los procesos de modernización en ciernes en la UBA y, en particular, en la Facultad de Ciencias Exactas y Naturales, las visiones sustentadas y los cambios impulsados por parte del grupo de profesores modernizadores de Medicina (y por parte de Lanari en particular) se centraron en lo concerniente a las acciones y proyectos de reformas en la enseñanza médica y la implementación y extensión del régimen full time en docencia e investigación. Esto quedó plasmado en la celebración del $1^{\circ}$ Congreso Argentino de Educación Médica, realizado por la Asociación Médica Argentina (AMA) en noviembre de 1957 (9).

Las ideas y acciones de reformas en torno a la enseñanza de la medicina, su organización asistencial y sus perfiles profesionales, impulsadas desde el plano regional e internacional, fueron compartidas por los modernizadores de la UBA, por el grupo de Houssay en la Facultad de Medicina y por los integrantes de la Tercera Cátedra de Clínica Médica. Todos ellos funcionaron como apoyos programáticos y acciones concretas que enmarcaron el proceso de construcción institucional del IIM.
Así, en el triple encuadre dado por la orientación común de los cambios impulsados en la enseñanza y la organización médica de posguerra, de las ideas de renovación y profesionalización científica propiciadas desde el rectorado de Risieri Frondizi y del itinerario de formación de Lanari, en el IIM se radicaron una variedad de servicios de atención, cursos de formación de grado y postgrado y diferentes grupos y líneas de investigación que dieron origen a más de tres generaciones de discípulos. Este conjunto de formas organizacionales e institucionales, de figuras y prácticas de investigación conformaron una tradición de investigación clínica que se institucionalizó y desarrolló en el espacio social delimitado por el IIM. Aún reconociendo esta construcción institucional -proceso del cual no solo formó parte Lanari, sino también otros colegas contemporáneos suyos- su "figura" desempeñó un rol diferencial, imprimiéndole el sello personal y constituyendo un pilar fundamental de la estructuración y evolución del ámbito de la investigación clínica.

\section{ACERCA DE LA INVESTIGACIÓN}

Este trabajo forma parte de mi tesis de doctorado (9) (c) y se concentra en la caracterización y el análisis de la intervención socio-profesional de Lanari en el medio local y en su itinerario de formación académica. Con ello, se intenta mostrar cómo su singularidad radicó en que supo crear un estilo de investigación propio (10), que conjugó elementos del "estilo de laboratorio", propio de la medicina experimental, con el "estilo de practicantes" de la clínica médica y del "estilo de investigación clínica", aprehendidos con sus maestros locales y en el extranjero, logrando reunir y resignificar prácticas de trabajo y culturas institucionales de los ámbitos experimentales y clínicos, de cara al medio clínico médico local. Este estilo le permitió hacerse un nombre y realizar su apuesta de inserción profesional en el medio clínico local, logrando diferenciarse de los meros investigadores experimentales y de los médicos practicantes, para inmediatamente después sellar la institucionalización de la investigación clínica médica, a partir de la creación del IIM en 1957. 
Esta investigación se llevó a cabo, entre 2007 y 2010, combinando el uso de fuentes orales y documentales. Con la realización de entrevistas en profundidad a miembros del Instituto de Investigaciones Médicas (contemporáneos de Lanari y discípulos de la primera, segunda y tercera generación), integrantes de la revista Medicina y de la Sociedad Argentina de Investigación Clínica, fue posible acceder a los discursos individuales y colectivos con rasgos profesionales comunes, como la especialidad, la experiencia asociativa y la pertenencia generacional. Dichos discursos fueron contrastados con fuentes y materiales estables tales como los legajos académicos de los investigadores (11-13) y los artículos científicos y de opinión de los investigadores, mayormente en la revista Medicina (13) pero también en otros canales locales de comunicación científica de la época, tales como Ciencia Nueva (14) y la Prensa Médica (15).

\section{ENTRENAMIENTO EXPERIMENTAL Y CLÍNICO: INSTITUCIONES Y REFERENTES LOCALES}

Alfredo Lanari, conocido entre sus amigos por el apodo de "Pipo", nació el 9 de febrero de 1910, en la ciudad de Mar del Plata, en el seno de una familia de clase media con una generación anterior de profesionales, por línea paterna (d).

En su período de formación de grado (1928-1934) tuvo participación en diferentes espacios académicos en los que, en forma temprana, desempeñó tareas de docencia e investigación, con inquietudes por el desarrollo tanto de la medicina experimental como de la clínica asistencial.

La primera tuvo a Bernardo Houssay como uno de los principales exponentes y precursores, ya que fue quien dio nacimiento a la fisiología, impulsando particularmente la investigación endocrinológica en el medio local (16). Lanari fue ayudante en la Cátedra de Fisiología, dirigida por Houssay desde 1929, y en ese marco eligió realizar su tesis de doctorado con él:

A Don Bernardo Houssay lo conocí cuando estaba cursando el segundo año de la Facultad de Medicina. Fui elegido para integrar una comisión especial que recibiría una enseñanza más intensa de la fisiología. Para los alumnos que tenían alguna vinculación familiar con la medicina, el nombre de Houssay era bien conocido. Tenía aureola de sabio, de alguien que se dedicaba por entero a la investigación y a la enseñanza. Todo lo que tenía vinculación con Houssay era en serio [...] ¿Qué fue lo que más nos impresionó de esta visión primera de Houssay? Que se encontrara siempre en su Instituto, ya sea en su escritorio o en los laboratorios enseñando e investigando. Una rara habilidad manual y quirúrgica que contribuía a que las demostraciones de los trabajos prácticos salieran siempre bien. (15 p.15)

La condición y modalidad de la dirección de Houssay implicaba la inscripción de los trabajos de tesis dentro de las líneas de interés y trabajo que se llevaban adelante en el Instituto de Fisiología. Ello formaba parte del sistema de montaje de trabajo ideado y practicado en ese marco institucional. Fue así que el tema de investigación sugerido por Houssay, retomando los propios intereses de Lanari, fue sobre la transmisión química de los impulsos nerviosos. Como en la mayor parte de las tesis que se hacían en el Instituto, Houssay no solo aconsejaba la elección del tema sino que, sobre todo al comienzo, él mismo realizaba los experimentos (14).

En Houssay se ha reconocido "un estilo de laboratorio" particular (17), conformado por un escaso uso de tecnología, una dinámica de trabajo intensivo, montaje de sistemas de experimentación, una aproximación holística a la fisiología y la elección deliberada de áreas de investigación endocrinológica en las que la competencia internacional era escasa. Si no todos, mucho de estos elementos tuvieron una influencia central en el itinerario científico posterior de Lanari en términos del entrenamiento que le brindó en fisiología, las habilidades y el estilo específico transmitidos, por los valores afectivos, personales y morales enseñados, así como también debido a la importancia otorgada a la construcción de generaciones de discípulos de cara a la reproducción ampliada de un grupo y de una institución científica (18).

La cultura clínica asistencial, propia de los "practicantes" (19), fue adquirida por Lanari a 
través de la figura de Mariano Castex. Al frente de la Primera Cátedra de Clínica Médica, entre 1918 y 1947, trabajó en el Hospital General de Agudos Carlos G. Durand y en el Hospital de Clínicas. Allí no solo ejerció la práctica médica sino que estuvo a cargo del dictado de cursos libres de clínica médica. Si bien abordó indagaciones de corte teórico y realizó algunos estudios experimentales, en forma predominante desarrolló una práctica clínica asistencial. Abarcó un amplio y diverso abanico de temas, tales como el estudio de la sífilis hereditaria, la parasitosis intestinal, los focos sépticos y las alergias, publicando trabajos de repercusión local e internacional (12).

Una vez graduado, en 1933, Lanari se desempeñó como médico asistente en el Servicio de Clínica Médica del Dr. Mariano Castex en el Hospital de Clínicas. Con él se entrenó en semiología y en la clínica asistencial, aprendiendo la relevancia de las rutinas de recorridos de sala, la minuciosa y sistemática observación clínica y la importancia de la dedicación profesional y personal en la atención y tratamiento de cada paciente. Mientras estas destrezas, adquiridas con Castex, fueron más tarde valoradas por Lanari, las atinentes a la investigación y su metodología de trabajo fueron más problemáticas:

\footnotetext{
Que yo no pensara como él en lo que respecta a la metodología de la investigación, no es otra cosa que 25 años de diferencia de edad y otro campo de investigación. Lo mismo dirían de mí los actuales investigadores adentrados en la biología molecular cuando me ven trabajando con perros. (15 p.16)
}

Lanari trabajó con Castex hasta 1947, momento en el cual Castex renunció a la universidad, en el marco de cesantías políticas y renuncias de académicos por las diversas tensiones mantenidas con el recién electo gobierno peronista $(1,14)$. Mientras con Castex el vínculo no perduró más allá en el tiempo, ni fuera del trabajo que desarrolló en su cátedra entre 1933 y 1947, con Houssay y Vaccarezza (de quien hablaré a continuación) mantuvo intercambios profesionales y personales muy fluidos, aun después de finalizado su trabajo en ambas cátedras. Junto con Houssay y Alfredo Bartolomé Lanari (padre), Raúl Francisco Vaccarezza fue la tercera figura de mayor trascendencia e influencia en la orientación y características de la formación científica de Lanari, en sus inicios y en su posterior desarrollo. Con él, a diferencia de Houssay y de Castex, mantuvo no solo un vínculo científico sino también un trato personal, amistoso y familiar (13).

Esta relación de mayor intimidad se forjó sobre la base del vínculo previo que Vacarezza ya tenía con Alfredo Bartolomé Lanari (padre): había sido su ayudante en la Cátedra de Física Médica y, fue uno de los médicos que lo acompañó en la nueva gestión, en calidad de Secretario de la Facultad entre 1919 y 1920 (durante el primer decanato) y 1927 y 1929 (durante el segundo mandato).

Vacarezza trabajó en el Instituto de Fisioterapia del Hospital de Clínica Médica y ocupó el puesto de practicante mayor en el Instituto Modelo de Clínica Médica. Luego inició su experiencia en el Hospital de Infecciosas "Dr. Francisco Javier Muñiz", donde adquirió reconocimiento científico por sus desarrollos de investigación, asistenciales y docentes sobre la tuberculosis. Fue profesor titular de la Cátedra de Patología y Clínica de la Tuberculosis, a partir de 1939, y estuvo al frente de la Dirección del Dispensario Antituberculoso y del Pabellón Koch, ambos también ubicados en el Hospital Muñiz (20).

Al finalizar su formación de grado, Lanari comenzó a trabajar en el Servicio de Enfermedades Infecciosas, ubicado en el Hospital Muñiz y dirigido por Vaccarezza:

\footnotetext{
Aunque comencé a concurrir al Muñiz antes del

39 [...] desde esa fecha hasta ahora, con largos lapsos en el extranjero, he estado vinculado a la Cátedra de Tuberculosis. He visto en ella lo que es una verdadera organización, lo que es la labor del profesor Vaccarezza, quien tenía que preocuparse desde dictar las clases hasta que me mandaran los perros a mi laboratorio experimental [...] Asistir durante estos quince años a las tres reuniones semanales, discutir los casos, sugerir tratamientos, inspirar investigaciones originales, leer todo lo que se publica en la cátedra. (21 p.57)
}

A diferencia de Castex, Vaccarezza sí era referenciado por el mismo Lanari como un promotor de la investigación clínica y también de la investigación experimental, particularmente en neumonología (15). 


\section{Entrenamiento con el grupo de Boston: el estilo de investigación clínica de Cannon, Luco y Rosenblueth}

Entre septiembre de 1938 y mayo de 1939, Lanari fue asistente voluntario en el Servicio del Profesor Minot, en Boston, donde conoció a Arturo Rosenblueth y a Joaquín V. Luco. Con ellos trabajó en la discusión y comparación entre la teoría eléctrica y teoría química sobre la transmisión sináptica del impulso nervioso, pero principalmente trazó lazos de suma importancia para la maduración de sus visiones sobre la medicina y su ejercicio profesional (22). Ambos fueron más tarde referentes de la neurofisiología: Rosenblueth fue director del Instituto de Estudios Avanzados del Instituto Politécnico de México, creador junto con Norbert Wiener de la cibernética, y Luco un referente de este campo de estudios en Chile. Lanari recordaba a Rosenblueth y Luco y sus influencias, siendo el primero "un amigo geográficamente lejano pero con quien estuve en comunicación constante hasta su muerte a fines de 1970" y el segundo, un "inseparable amigo en Boston, cuya decisión de volver a trabajar full time en Chile, influyó en mí, apartándome del esquema clásico del clínico con dedicación parcial para impulsarme a la rigurosidad del docente e investigador con dedicación exclusiva" (23 p.36-37).

Cuando Lanari conoció a ambos en Boston, Walter Cannon era la otra figura que estaba detrás de ellos. Una de las más reconocidas personalidades científicas de la medicina mundial, desde su servicio en la Primera Guerra Mundial hasta su muerte a fines de la Segunda, Cannon fue uno de los precursores en el campo de la Fisiología, ocupando el cargo de jefe de fisiología de la Harvard Medical School entre los años 1920 y 1940 (22).

También allí Lanari realizó una estadía de perfeccionamiento, entre 1938 y 1939, donde trabajó junto a Cannon. Esta relación y las entabladas con Luco y Rosenblueth constituyeron las experiencias más importantes para Lanari, pues a partir de allí sedimentó su visión singular respecto a romper la dualidad entre la medicina clínica y la experimental (de laboratorio) y afianzó su inclinación por la investigación. Cannon era un impulsor de la conjunción de ambas, en particular, de la fisiología y su aplicación en la clínica (24):

Trabajé con Cannon y Rosenblueth no más de ocho o nueve meses, se publicaron algunos trabajos y después supe exactamente lo que quería hacer. Volví a Buenos Aires con la idea de que me dedicaría en forma full time a la investigación clínica. (14 p.26)

Efectivamente Cannon reforzó la vocación por la fisiología que Lanari había recibido de Houssay y contribuyó a que la llevara -con sus métodos, prácticas e ideas- a la medicina clínica. Luego de su estadía en Harvard, Lanari desarrolló más formalmente su predilección por la fisiopatología pulmonar clínica. Más tarde, la influencia de esos referentes iba más allá de la elección de una línea de investigación. La ideología y el estilo de Cannon, Luco y Rosenblueth se verían plasmados en el proyecto institucional y cognitivo del IIM.

\section{CONFORMACIÓN DE UN ESTILO DE INVESTIGACIÓN PROPIO}

Al llegar a Buenos Aires, en 1939, no teniendo la posibilidad de hacer investigación clínica full time dado que no existían posiciones con tales dedicaciones para un clínico en la Facultad de Medicina, dividió su tiempo de trabajo entre la clínica y la medicina experimental: por la mañana, en forma parcial, con Mariano Castex, como adscripto a la Cátedra de Clínica Médica, y por la tarde, en el Instituto de Fisiología.

Al poco tiempo reemplazó este trabajo que era ad honorem -aunque nunca dejó de ir al Instituto y de tener intercambios frecuentes con Houssay- e ingresó al Hospital Muñiz, donde funcionaba la Cátedra de Patología y Clínica de la Tuberculosis, a cargo de Vaccarezza quien le otorgó un puesto con un sueldo que, aunque no fuera full time, le permitía vivir: fue nombrado Jefe Interino de Investigaciones Fisiopatológicas de la Cátedra. A partir de esta incursión, comenzó a desarrollar líneas de trabajo en temas sobre fisiología respiratoria. Estos se sumaron a sus primeras líneas de investigación en el campo de la neurofisiología y de las enfermedades 
neuromusculares (siendo la miastenia gravis la que recibió su mayor atención), las cuales nunca fueron abandonadas si bien fueron interrumpidas o discontinuadas en distintos momentos de su carrera (14).

Sumado a estos desempeños, también ocupó el cargo de médico en la Dirección General Impositiva. Esto le implicaba una carga de trabajo importante pero necesaria para redondear un ingreso que le permitiera vivir, ya que era dificultoso hacerlo solo con las dedicaciones docentes parciales en la universidad y el hospital.

Asimismo, al poco tiempo de su regreso, en octubre de 1939, junto a un grupo de médicos que se habían formado con Houssay pero su orientación de investigación no se reducía al campo experimental (fisiología) sino a su aplicación clínica, fundó la revista Medicina, a cuyo comité editorial perteneció hasta su muerte, en 1985. La publicación y difusión de este tipo de trabajos de investigación clínica, requería un órgano específico y con tal fin fue que se creó la revista Medicina:

Yo diría que el grupo fundamental que está con Houssay es Braun Menéndez, Foglia, Lanari. Esos sujetos se fueron volcando bastante hacia el estudio del ser humano directamente, son los mismos que, en un momento dado, cuando empiezan a terminar sus trabajos y querer divulgarlos, encuentran que, en la Argentina, no hay una sociedad apta para publicar sus trabajos [...] De modo que, cuando pasan los años y ya aparecimos nosotros, que somos "las manos de ellos" (ellos ponían la cabeza, nosotros podemos decir que poníamos las manos), en el año 1960, surgió la Sociedad Argentina de Investigación Clínica. Pero mucho antes, ese mismo grupo se reúne para publicar la revista Medicina, es en el 39; son todos del grupo de Houssay. (e)

La revista había nacido por inspiración de Lanari y otros miembros, quienes en su mayoría no superaban los 30 o 35 años de edad y conformaban, por eso, la nueva generación de médicos formados en los años posteriores a la Reforma Universitaria de 1918: Alberto Taquini, Osvaldo Fustinoni, Venancio Deulofeu, Mario Brea, Alfredo Pavlovsky, entre los principales exponentes (25).
Al escribir las palabras iniciales del primer número, Lanari resaltaba que la plataforma de la revista necesitaba "dar a la familia médica argentina una revista de medicina general cuya organización esté fundamentada en la calidad de los artículos originales" (25 p.1). Esta misión se materializaba en una de las tres secciones que estructuraban la revista: una primera, destinada a la publicación de trabajos originales y casuísticas, siempre que fueran "breves y contemplaran un aporte" (en términos de nuevos conocimientos y no una mera descripción de un caso clínico); la segunda sección tenía por objetivo "poner de relieve nuevas adquisiciones en cualquier sector de la medicina"; y la tercera, se reservaba para los trabajos de revisión crítica de libros locales y extranjeros. Un cuarto espacio fue conformado por las editoriales (25 p.1).

En octubre de 1943, en el marco del proceso de cesantías de profesores universitarios que inició el gobierno de facto establecido en el país, Lanari renunció a los cargos que tenía en la cátedra de Castex y en la de Vaccarezza. El alejamiento de la universidad duró un lapso de tiempo relativamente corto, ya que al cabo de dos años, en mayo de 1945, Lanari se reincorporó a la Facultad. Entonces, obtuvo la designación de Jefe de Laboratorio Experimental de la Cátedra de Patología y Clínica de la Tuberculosis y comenzó a dictar su primer curso de "Docencia complementaria de Clínica Médica", en el servicio de Castex. Asimismo, en septiembre de ese año fue designado médico agregado honorario con servicio en la Sala V del Hospital de Clínicas (24).

Durante el período de 10 años que se extendió hasta su designación como titular docente e investigador full time de la Tercera Cátedra de Clínica Médica y de la dirección del IIM en el Hospital Tornú, Lanari continuó desarrollando y ampliando actividades de formación en el ámbito local y en el extranjero.

Desde 1947, dictó Fisiopatología de la Respiración en el curso de perfeccionamiento para médicos tisiólogos a cargo de Vaccarezza y continuó como Jefe de Laboratorio Experimental en su cátedra, sumando su colaboración, como auxiliar, en el dispensario antituberculoso. A su vez, obtuvo por concurso la designación de profesor adjunto de la Primera Cátedra de Clínica Médica en el mes de junio de 1950. 
En 1949 lo dejaron cesante en el puesto de médico que tenía en la Dirección General Impositiva dado que se negó a firmar una solicitada oficial, promovida por el gobierno peronista (14). Por eso, entre 1949 y 1951 solo ejerció la medicina privada, asistiendo pacientes en su consultorio y en una clínica: el Instituto del Tórax, junto a Vaccarezza y a otros miembros del grupo del Hospital Muñiz (14).

Aquel episodio de 1949 se sumaba al iniciado en 1943 con las cesantías masivas de profesores en la universidad. En 1947 se puso en vigencia la Ley Universitaria 13.031 que suprimía el Estatuto de la Reforma Universitaria de 1918, anulándose la autonomía, el gobierno tripartito y la participación estudiantil. Ante esta acumulación de condiciones adversas para su desarrollo profesional y ante la oferta de un puesto de trabajo y de investigación en EE.UU., decidió irse del país: "Ya estaba bastante harto de la situación política del país y tenía dificultades en mi trabajo; me ofrecieron un puesto y me fui" (14 p.27).

\section{Segunda etapa en el exterior en el National Jewish Hospital, Denver}

Entre enero y mayo de 1951, con una beca de la Fundación Rockefeller, Lanari realizó una estadía de investigación en EE.UU., en la University of Colorado, en Denver. Allí se concentró en el estudio de los métodos empleados en la exploración funcional del aparato respiratorio (26). Luego, entre fines de 1951 y julio de 1952, fue designado por la Organización Mundial de la Salud (OMS) como Asesor en Fisiopatología Cardiopulmonar en Guayaquil, Ecuador.

Al año siguiente, regresó a la Argentina y fue designado profesor adjunto asistente en la Tercera Cátedra de Clínica Médica y, un mes más tarde, docente ad honorem del curso de perfeccionamiento en tisiología en la Cátedra de la Tuberculosis. En este período, aprovechó su tiempo en la preparación de su tesis de profesorado "El problema general de injertos y transplante de órganos", aprobada en 1954 (24).

Luego, en ese mismo año se volvió a ausentar del país, radicándose como profesor asistente de medicina en la University of Colorado, en Denver, hasta 1956. Allí trabajó en el National Jewish Hospital, asociado a dicha universidad, donde fue Jefe del Laboratorio Cardiopulmonar y Profesor Asistente. Durante esos años de trabajo en Denver, Lanari invitó a trabajar con él a varios médicos jóvenes que luego formaron parte del staff del IIM (27).

$\mathrm{Su}$ regreso y establecimiento en la Argentina se debieron a las condiciones profesionales favorables que vislumbró en el nuevo escenario nacional y científico abierto después de 1955, con el proceso de renovación y reconstrucción universitaria antes referido (28). En este marco, se abrió un concurso para profesor titular de Clínica Médica, a mediados de 1956. Este suceso en concreto fue lo que definió su regreso, presentándose al mismo con la exigencia de que la dedicación fuera full time.

Esta modalidad de trabajo, núcleo central de los ideales profesionales, que Lanari llevó delante desde entonces, había sido aprehendida primero con Houssay y luego con Rosenblueth, Luco y Cannon. La singularidad y novedad de estos últimos tres fue colocar el full time en la incumbencia profesional del investigador clínico:

Junto a ellos me di cuenta de que la única forma
de hacer investigación en serio era haciéndola full
time. Como teoría, por lo menos, uno debe preten-
der que el investigador se dedique a la investiga-
ción clínica, vea y asista a los enfermos en el
mismo lugar de trabajo, y que no tenga preocupa-
ciones que requiere la práctica privada. En reali-
dad, ser full time es una posición mental. (14 p.27)

En estas reflexiones sobre la naturaleza y funciones del full time médico investigador, destacaba también su visión sobre la plataforma organizacional y física adecuada para dicha práctica: la de una ecología común entre atención e investigación en la clínica. Esta visión se plasmaría más tarde en una cultura organizacional de trabajo integral de asistencia, investigación y enseñanza en el IIM.

\section{Regreso y radicación: titular full time de la Tercera Cátedra de Clínica Médica}

En octubre de 1956, de vuelta en Argentina, obtuvo la designación de profesor full 
time titular de la Tercera Cátedra de Clínica Médica, convirtiéndose en el primer profesor de Clínica Médica con tal dedicación en la Facultad de Medicina de Buenos Aires (f). Para ese cargo inmediatamente pidió licencia, hasta febrero de 1957, con el fin de viajar a Denver, en donde había dejado inconclusas actividades de investigación en el National Jewish Hospital, y de docencia en la University of Colorado (24).

A su regreso, en julio de 1957, ante la apertura del IIM, cuya dirección le fue ofrecida, solicitó serlo siempre y cuando fuera designado full time, aunando dicho cargo con la titularidad obtenida un año antes. La comisión permanente, encabezada por Houssay, encargada de estudiar las medidas tendientes al fomento y financiación de la investigación científica en la Facultad de Medicina, aceptó ese pedido, declarándolo en condiciones de dedicación exclusiva y juntando ese cargo de dirección con el cargo de profesor titular de la Tercera Cátedra de Clínica Médica. Desde allí, se lanzó a liderar la conformación inicial del IIM, reclutando colegas de dicha cátedra, al grupo que había trabajado con él en el Hospital Muñiz y en el Hospital de Clínicas y a otros investigadores que se habían perfeccionado en el exterior (24).

La experiencia y el recorrido que Lanari iniciaría en adelante, como un investigador formado, no fue sino sobre la base de la integración y resignificación de los legados de las tradiciones clínicas y experimentales locales y extranjeras que transitó en estos años de formación. Dichos legados consistieron en específicas modalidades de ejercicio de prácticas asistenciales, de investigación y de enseñanza y en la construcción y el sostenimiento de ambientes institucionales y modelos organizacionales afines al desarrollo integral de tales actividades.

\section{OBSERVACIONES FINALES}

Si prestamos atención a la evolución de las líneas de investigación abiertas y desarrolladas por Lanari, como a sus diferentes inserciones institucionales durante su formación, observamos cómo los estilos de investigación de la vertiente experimental de Houssay y las prácticas y visio- nes sobre la asistencia a pacientes de la clínica médica, representadas por Vaccarezza y Castex, contribuyeron a forjar el estilo Lanari. Aquello que no tomó de la figura pionera en la fisiología argentina, y sí en cambio de sus maestros clínicos, fue la alta valoración por las actividades asistenciales: el paciente y su enfermedad como eje orientador del interés médico y de la formulación de problemas de investigación.

Por eso, la singularidad de Lanari radicó en que el estilo de los practicantes aprendido con Castex, y el de investigación clínica con Vaccarezza, lo conectó con el estilo de laboratorio de Houssay: no solo estimaba que las actividades asistenciales eran relevantes en sí mismas sino siempre que fueran utilizadas como insumos insustituibles para realizar investigación clínica, es decir, producir conocimiento original orientado a la resolución de (o inspirados en) problemas de la salud humana. Esta conjunción de prácticas y culturas institucionales adquirió un modo singular en Lanari: su modalidad de desempeño full time en el ámbito clínico universitario y hospitalario. Éste fue el legado que asimiló de sus maestros Luco, Cannon y Rosenblueth y que resignificó de cara a sus predecesores del ámbito de la medicina clínica local, entre quienes nunca antes se habían despertado tales tipos de vocaciones. Fue entonces la conjunción de las culturas y prácticas experimentales y clínicas en una modalidad de trabajo full time el sello y la originalidad del itinerario de formación de Lanari.

Más tarde, su trayectoria, visiones y prácticas fueron convergentes con un contexto político académico local y otro disciplinar, afines y favorables a su materialización institucional. Por un lado, el de la llamada modernización académica o renovación de la universidad argentina posterior a 1955, que promovió centralmente la profesionalización del rol investigador-docente y, por el otro, el fomento del quehacer investigativo experimental desde los entramados financieros y promotores norteamericanos y franceses, fortaleciendo la denominada "medicina científica" (2). Estos procesos posibilitaron la creación del IIM y la institucionalización del estilo Lanari que daría cauce al desarrollo de una tradición de investigación propia, desarrollada a lo largo de 20 años. Pero este "clima de época" favorable, así como la creación del IIM, son objetos de reflexión que 
escapan a los límites y metas planteadas en este trabajo; su mención obedece a dejar señalado al menos el rumbo que más tarde tomaría su trayectoria individual en el marco de un proyecto institucional materializado en el IIM.

\section{NOTAS FINALES}

a. La universidad argentina se originó y evolucionó al calor de la tradición profesional napoleónica, estructurándose básica y fundamentalmente como un espacio de reproducción y circulación de saber. Así, delineó para sí un perfil profesionalista que tuvo por función principal expedir títulos habilitantes para el ejercicio de las profesiones liberales clásicas, reservando un lugar subsidiario para las actividades de producción de conocimiento original (7). Las transformaciones que el proceso de la Reforma Universitaria de 1918 introdujo en la universidad argentina redundaron, principalmente, en aspectos organizativos de su gobierno y de la carrera académica y, si bien implicó una revalorización de la universidad como ámbito de investigación, en ello encontró sus contradicciones y límites más profundos, conservando el patrón profesionalista que la signó desde su origen (8). En perspectiva con este encuadre precedente, la fase histórica abierta en la Universidad de Buenos Aires, entre 1955 y 1966, valorada en términos de la "reconstrucción universitaria" (1), adquirió un significado muy peculiar en cuanto se otorgó, material y simbólicamente, un lugar de renovada importancia a la producción de conocimiento original. Esto se tradujo en la creación y multiplicación de plazas de dedicación exclusiva (full time) a la actividad de investigación y docencia, lo cual dio paso a la profesionalización de tales actividades y al establecimiento y la apertura de concursos para la provisión y ocupación de dichos cargos. En este cuadro de situación, la discusión de fondo, impulsada por los renovadores como Risieri Frondizi, consistía en el debate acerca de la "función social" de la universidad cuya primera función se postulaba fuera la de investigación -junto con la de enseñanza-; luego también la de formación de profesionales y de extensión social. Estos sentidos atados a la función social de la universidad recuperaban el ideario reformista, en su tensión más constitutiva -autonomía y extensión- dándole un nuevo encauzamiento en el nuevo contexto de reforma académica abierto a partir de 1955. Desde una mirada más amplia y general, no puede eludirse el marco de ideas y procesos en el cual se encuadró este conjunto de acciones y proyectos de renovación en la universidad argentina, que se orientaron a otorgarle a la ciencia y a la universidad un lugar de renovada significación en la sociedad. Durante la segunda posguerra se originó una nueva fase en las relacio- nes entre la ciencia y el Estado. En los países centrales, este comenzó a otorgarle a la ciencia y a la tecnología un lugar de nuevo privilegio en sus agendas, aumentando sus recursos, creando nuevas instituciones dedicadas a la investigación así como también organismos nacionales y regionales especializados, tales como la Organización de las Naciones Unidas para la Educación, la Ciencia y la Cultura (UNESCO) y el Centro de Estudios para América Latina (CEPAL), destinados a la promoción y regulación de la ciencia y la tecnología. Esta fue una tendencia seguida en los países en desarrollo, bajo el clima de ideas y acciones denominado "desarrollismo", donde comenzaron a crearse, al promediar la década de 1950, Consejos Nacionales de Investigación -en 1958, se creó en el país el Consejo Nacional de Investigaciones Científicas y Técnicas (CONICET)- así como también Institutos Nacionales abocados a la investigación, gestión y promoción de actividades científicas y tecnológicas; en 1956 se creó el Instituto Nacional de Tecnología Agropecuaria (INTA) y, un año más tarde, el Instituto Nacional de Tecnología Industrial (INTI).

b. Inaugurado en 1919, el Instituto de Fisiología y su director, Bernardo Houssay, fueron centrales para la consolidación de la medicina experimental en la Argentina y de la fisiología y la endocrinología en el concierto mundial médico (por cierto, su gravitación en la política científica argentina no fue menos importante). Para los años 40, el Instituto era una parte central de la Facultad de Medicina, con un staff de 130 instructores que enseñaban fisiología, bioquímica y biofísica a más de 800 alumnos. Si bien trabajó en varias líneas de investigación, su mayor inversión fue sobre la relación entre la hipófisis y la diabetes, por lo cual obtuvo el Premio Nobel en Medicina en 1947, precisamente por el descubrimiento del papel de la hormona del lóbulo anterior de la hipófisis sobre el metabolismo de los hidratos de carbono (6). Desde este Instituto se proyectó el "grupo de Houssay". En el año 1958, Florencio Escardó fue nombrado como decano normalizador de Medicina, apoyado desde el Consejo Directivo por Eduardo Braun Menéndez, Eduardo De Robertis, Alfredo Lanari, Mario Brea, Eduardo Manzini, Armando Parodi, Venancio Deulofeu.

c. Este artículo está basado en "Conformación de la tradición: Alfredo Lanari, la construcción de un estilo de investigación propio" (9). 
d. Su socialización primaria resulta significativa si se considera el peso y el lugar que ocupó su padre en la medicina argentina en la década de 1920. Alfredo Bartolomé Lanari (padre) dirigió el Instituto de Fisioterapia, donde dictó Física Médica. Fue elegido Decano de la Facultad de Medicina en mayo de 1919 -y posteriormente en 1927 hasta 1929-, dando lugar con su mandato de tres años a un conjunto de transformaciones muy relevantes en la enseñanza de la medicina: la implantación efectiva de la docencia libre en la Facultad, la limitación de la edad dentro de la cual los profesores estaban en condiciones para dictar clases y la modificación del plan de estudios. Entre los principales cambios, el nuevo plan redujo la enseñanza de la anatomía descriptiva y realzó el lugar de las ciencias básicas con la creación del Instituto de Fisiología
(11). Esta modalidad de trabajo y compromiso con la investigación y la docencia como la participación y actividad política académica en el ámbito de gobierno de la Facultad evidentemente operaron como modelos de desempeño profesional, internalizados positivamente por su hijo, quien también modeló su trayectoria dándole significación a estas tres esferas de acción, desempeñándolas en una modalidad full time.

e. Barousse Amadeo, investigador del Instituto de Investigaciones Médicas, Sección Neumonología, $3^{\circ}$ Jefe. Entrevista personal, 4 de marzo de 2009.

f. La Tercera Cátedra había estado en manos de Alberto Taquini hasta 1955. Para el concurso de titular con dedicación exclusiva celebrado en 1956 se presentó solamente Lanari y lo ganó.

\section{REFERENCIAS BIBLIOGRÁFICAS}

1. Halperín Donghi T. Historia de la Universidad de Buenos Aires. Buenos Aires: Eudeba; 1962.

2. Gaudillière JP. Inventer la biomédicine: La France, I' amerique et la production des savoirs du vivant (1945-1965). Paris: La Découverte; 2002.

3. Bustíos Romani C. Notas sobre la historia de la educación médica 1933-1980: Segunda parte. Acta Médica Peruana. 2003;20(3):133-149.

4. García JC. La educación médica en la América Latina. Washington DC: OPS; 1972.

5. Flexner A. Medical Education in the United States and Canada Bulletin Number Four (The Flexner Report) [Internet]. New York: The Carnegie Foundation for the Advancement of Teaching. Carnegie Bulletin No. 4 [citado 2 dic 2011] http://www.carnegiefoundation.org/sites/default/fil es/elibrary/Carnegie_Flexner_Report.pdf

6. De Asúa M. La formación del médico en la República Argentina: Análisis histórico-sistemático del perfil curricular de las Facultades de Medicina de la Argentina desde su creación hasta 1982. [Tesis de doctorado]. Buenos Aires: Facultad de Medicina, Universidad de Buenos Aires; 1984.

7. Romero JL. El ensayo reformista. En: Situaciones e Ideología en Latinoamérica. Buenos Aires: Sudamericana; 1986. p. 172-198.
8. Buchbinder P. El movimiento reformista de 1918: Una perspectiva desde la historia interna de la Universidad de Buenos Aires. Estudios Sociales. 2000; 10(19):37-63.

9. Romero L. Conformación de la tradición: Alfredo Lanari, la construcción de un estilo de investigación propio. En: Conformación y desarrollo de una tradición de investigación clínica médica: Alfredo Lanari y el Instituto de Investigaciones Médicas (1957-1976) [Tesis de doctorado] Buenos Aires: FLACSO Argentina; 2010. p. 91-124.

10. Gayon J. On the uses of the category of style in the history of science. Philosophy \& Rhetoric. 1999; 32(3):233-246.

11. Universidad de Buenos Aires. Facultad de Medicina. Legajo de Lanari Alfredo Bartolomé $N^{\circ}$ 8719. [Material de Archivo]. Buenos Aires: Archivo General, Biblioteca Central.

12. Universidad de Buenos Aires. Facultad de Medicina. Legajo de Castex Mariano No 33920, tomos I y II. [Material de Archivo]. Buenos Aires: Archivo General, Biblioteca Central.

13. Lanari A. Profesor Don Raúl F. Vaccarezza. Medicina. 1981;41:390-391.

14. Investigación en clínica médica: Reportaje a Alfredo Lanari. Ciencia Nueva. 1970;(3):26-33.

15. Lanari A. Discurso pronunciado por Alfredo Lanari. La Prensa Médica Argentina. 1971;67:1418. 
16. Buch A. Forma y función de un sujeto moderno: Bernardo Houssay y la fisiología argentina (1900-1943). Buenos Aires: Universidad Nacional de Quilmes; 2006.

17. Cueto M. Laboratory styles in Argentine Physiology. Isis. 1994; 85: 228-246.

18. Lanari A. Homenaje a Houssay: Conferencia pronunciada en el XI Congreso Latinoamericano de Ciencias Fisiológicas, el 29 de julio de 1973. En: Martín R, Barcat JA, Molinas F, compiladores. Alfredo Lanari. Vocación y convicción. Reflexiones sobre la investigación, el futuro de la medicina y otros escritos. Buenos Aires: Fundación Alfredo Lanari; 1995. p. 147-154.

19. Camargo K. The thought style of physicians: strategies for keeping up with medical knowledge. Social Studies of Science. 2002; 32(5-6):827-855.

20. Universidad de Buenos Aires. Facultad de Medicina. Legajo de Vaccarezza Raúl № 34.030, tomo I. [Material de Archivo]. Buenos Aires: Archivo General, Biblioteca Central.

21. Lanari A. Tercera Cátedra de Clínica Médica: Clase Inaugural. En: Martín R, Barcat JA, Molinas F, compiladores. Alfredo Lanari. Vocación y convicción. Reflexiones sobre la investigación, el futuro de la medicina y otros escritos, Buenos Aires: Fundación Alfredo Lanari; 1995. p. 55-61.
22. Wolfe E, Barger C, Benison S. Walter B Cannon: Science and Society. Boston: Medical Library; 2000.

23. Lanari A. Discurso del Académico recipiendario Dr. Alfredo Lanari. Boletín de la Academia Nacional de Medicina; 1973(51):31-46.

24. Universidad de Buenos Aires. Facultad de Medicina. Legajo de Lanari Alfredo No 51456. [Material de Archivo]. Buenos Aires: Archivo General, Biblioteca Central.

25. Lanari A. Palabras iniciales. Medicina. 1940;1(1):1-3.

26. The Rockefeller Foundation. Annual Report 1950. New York: The Rockefeller Foundation; c2003.

27. Santas A. Semblanza de los premiados por Andrés A. Santas. La Prensa Médica. 1980(67):1-14.

28. Prego C. La gran transformación académica y su política a fines de los años 50: El proyecto de reorganización institucional y los inicios del debate del cientificismo en la Universidad de Buenos Aires. En: Prego C, Vallejos O, editores. La construcción de la ciencia académica: actores, instituciones y procesos en la universidad argentina del siglo XX. Buenos Aires: Biblos; 2010. p. 133-159.

\section{FORMA DE CITAR}

Romero L. Alfredo Lanari, un estilo de investigación clínica. Salud Colectiva. 2012;8(2):69-80.

Recibido el 20 de junio de 2011

Versión final presentada el 14 de septiembre de 2011

Aprobado el 3 de octubre de 2011 